\title{
Sliding Manipulation of Rigid Bodies on a Controlled 6-DoF Plate
}

\author{
Thomas H. Vose, Paul Umbanhowar, and Kevin M. Lynch
}

\begin{abstract}
We model the full dynamics of a rigid part in three-point frictional sliding contact with a flat rigid 6-degree-offreedom plate. Given a periodic plate motion and the geometric, inertial, and frictional properties of the part, we define an asymptotic twist field mapping each part configuration to a unique part twist (linear and angular velocity). Asymptotic twist vectors in the field approximate the part's cycle-averaged twist at each configuration and are independent of time or the system's initial state. Simulations and experiments show that the trajectory of the part's configuration as it slides on the plate is well described by the field. With the ability to program arbitrary plate motions, part manipulation reduces to finding plate motions that generate asymptotic twist fields to accomplish desired tasks. Several simple fields useful for manipulation tasks (e.g., sensorless part alignment) are verified in simulation and experiment. For the special case of a rigid part with infinitesimal thickness, we show that the part's cycle-averaged twist for any configuration asymptotically converges to a unique asymptotic twist vector.
\end{abstract}

\section{INTRODUCTION}

One method of manipulating an extended part on a planar surface is to create a smooth force field on the surface. The part's trajectory is obtained by integrating the forces and moments over the contact area. If the fields are programmable, the surface can perform various automation and assembly tasks on one or multiple parts without grasping or sensing them. Research in this area has primarily focused on designing fields that automatically position and orient planar parts [1], [2], [3], [4], [5], [6], [7], [8].

In practice, generating continuous force fields on a surface is difficult. Discrete force fields have been modeled as arising from planar arrays of actuators, such as MEMS elements [2] or rolling wheels [5], [7]. Although these are often approximated as continuous fields, undesirable part behavior may result if the actuators are not packed densely enough [5], [7].

Continuous force fields have been modeled as arising from frictional forces acting on parts sliding on vibrating plates [2], [9], [10], and pressure-induced forces acting on parts due to controlled air flow [8]. The models for these systems tend to be idealized-e.g., in vibratory systems the part is often modeled as a point mass [9] or an extended planar object with linear pressure distribution [10] - and the predicted force fields are more qualitative than quantitative.

Motivated by the continuous fields and small number of actuators of vibrating plates, this paper examines friction-based manipulation of extended rigid parts in three-point contact with a flat, rigid, 6-degree-of-freedom (DoF) plate undergoing

This work was supported by NSF grants IIS-0964665 and CMMI-0700537. T. H. Vose, P. Umbanhowar, and K. M. Lynch are with the Department of Mechanical Engineering, Northwestern University, Evanston, IL 60208 USA. K. M. Lynch is also affiliated with the Northwestern Institute on Complex Systems. \{t-vose, umbanhowar, kmlynch\}@northwestern.edu

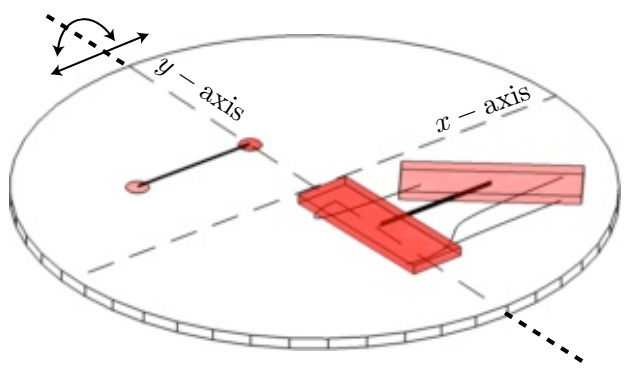

(a)

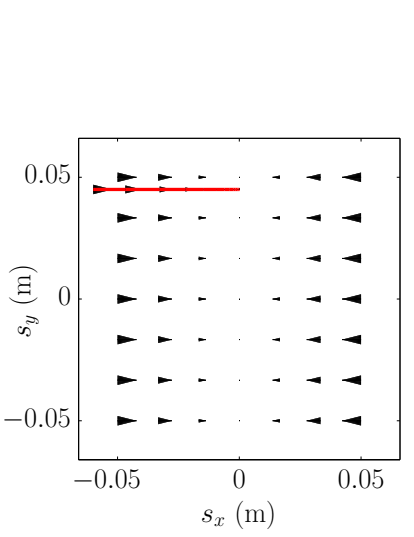

(b)

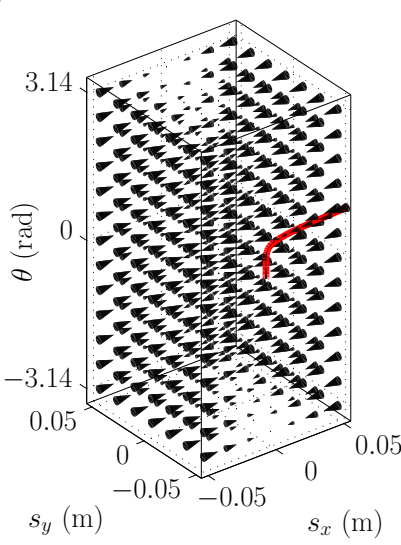

(c)

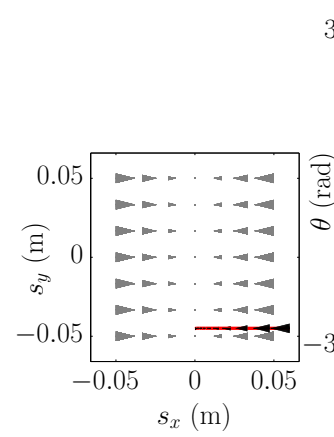

(d)

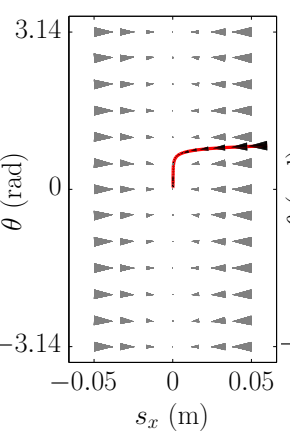

(e)

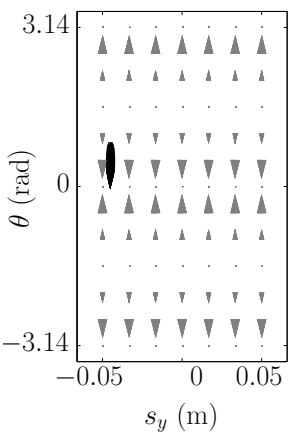

(f)
Fig. 1. Positioning and orienting parts to a line without sensors. (a): Overhead view of a rigid vibrating plate with a point part (disk) and a rigid part (cuboid). The rigid part has three "feet" in point contact with the plate. Both parts start from rest on opposite sides of the plate and converge to a common line coincident with the plate's $y$-axis. The thick black curves denote the path of the center of mass for each part. The thin black curves denote the paths of the rigid part's three contact feet. The plate motion consists of symmetric rotation about the $y$-axis coupled with symmetric translation along the $x$-axis (see black arrows above the plate). Section IV-C has exact part and plate information. (b): The trajectory of the point part's configuration $\left(s_{x}, s_{y}\right)$ is described by an integral curve (red line) of an asymptotic velocity field on $\mathbb{R}^{2}$. Field vectors at configurations along the trajectory are plotted every 0.25 seconds. (c): The trajectory of the rigid part's configuration $\left(s_{x}, s_{y}, \theta\right)$ is described by an integral curve (red line) of an asymptotic twist field on SE(2). (d)-(f): Trajectory and field vectors in (c) projected onto the $s_{x}-s_{y}, s_{x}-\theta$, and $s_{y}-\theta$ planes. Field vectors along the trajectory are plotted every 0.25 seconds in black. Gray field vectors are projections of the spatially distributed vectors in (c). Zoom in for more detail. 
controlled periodic vibration. We show that the part's trajectory is naturally associated with a continuous twist field (i.e., a generalized velocity field) on the part's three-dimensional configuration space-not a force field on the plate's twodimensional surface. We call this field an asymptotic twist field because the part's cycle-averaged twist at any configuration asymptotically converges to it regardless of initial conditions. This result extends our previous work in which we showed that the trajectory of a point part on a periodically vibrating plate is described by an asymptotic velocity field on the part's twodimensional configuration space [11], [12], [13]. The extension from velocity field to twist field is illustrated in Fig. 1. The plate motion depicted in Fig. 1(a) generates the asymptotic velocity field shown in Fig. 1(b) for the point part; it also generates the asymptotic twist field shown in Fig. 1(c) for the rigid part. Both fields automatically move the parts so that their centers of mass are over the plate's $y$-axis; the asymptotic twist field for this particular rigid part also orients it such that its principal axis is aligned with the plate's $y$-axis. This is just one of many sensorless manipulation tasks that can be done on a vibrating plate by exploiting an asymptotic twist field.

Assumptions must always be made about the contact interface when extending from point parts to parts in distributed frictional contact with the plate (e.g., compliant contact, rigid contact, imposed pressure distribution). Here we model the system as rigid bodies in three-point contact because the normal forces and part accelerations can be solved for analytically (and uniquely) without additional assumptions about the compliance of the contact interface or the form of the pressure distribution.

The contributions of this paper can be summarized as: a derivation of the equations of motion of a rigid body in three-point frictional sliding contact with a moving surface (Section III); extending the concept of asymptotic velocity fields on $\mathbb{R}^{2}$ for point parts to asymptotic twist fields on $\operatorname{SE}(2)$ for rigid bodies (Section IV); simulations and experiments demonstrating that asymptotic twist fields accurately describe part motion (Sections IV and V); and an outline of a proof of asymptotic convergence to the asymptotic twist field for a simplified version of the dynamics (Appendix).

\section{Coordinate Frames And Notation}

Throughout this paper, scalars and points in $\mathbb{R}^{3}$ are written in lowercase italic, vectors are written in lowercase bold, and matrices are written in uppercase bold. Pre-superscripts are used to indicate the coordinate frame in which a vector quantity (or a point represented as a vector) is expressed (e.g., $\mathcal{A}_{\mathbf{x}}$ is the location of point $x$ in $\mathcal{A}$ coordinates). The rotation matrix that transforms a vector in $\mathbb{R}^{3}$ from $\mathcal{A}$ to $\mathcal{B}$ coordinates is written ${ }_{\mathcal{A}}^{\mathcal{B}} \mathbf{R} \in S O(3)$. The angular velocity of frame $\mathcal{A}$ with respect to frame $\mathcal{B}$ is written $\omega_{\mathcal{A} / \mathcal{B}}$.

Fig. 2 gives an overview of the notation. Let $s$ represent the location of the center of mass of the part, $p$ represent the location of the center of the upper surface of the plate, $c_{1}, c_{2}$, and $c_{3}$ represent the locations on the part in contact with the

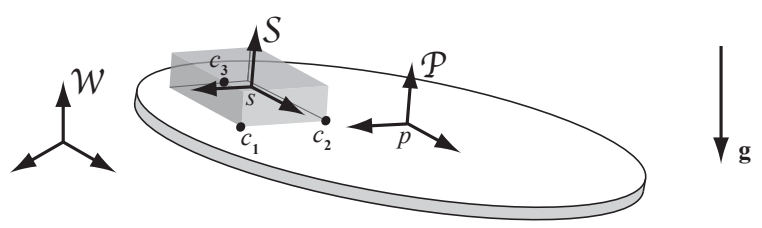

Fig. 2. Part-plate schematic with coordinate frames. Coincident with the $\mathcal{P}$ and $\mathcal{S}$ frames (but not shown) are the $\mathcal{P}^{\prime}$ and $\mathcal{S}^{\prime}$ frames, respectively.

plate, and $c_{1}^{*}, c_{2}^{*}$, and $c_{3}^{*}$ represent the locations on the plate in contact with the part.

Let $\mathcal{P}$ be a non-inertial frame fixed to the plate with origin $p$. Let $\mathcal{W}$ be a stationary inertial world frame that coincides with $\mathcal{P}$ when the plate is in the home (horizontal) configuration. The $z$-axis of $\mathcal{W}$ is opposite the gravity vector g. Let $\mathcal{S}$ be a non-inertial frame fixed to the sliding part with origin $s$. The $z$-axes of $\mathcal{S}$ and $\mathcal{P}$ are parallel. Let $\mathcal{P}^{\prime}$ and $\mathcal{S}^{\prime}$ be inertial frames that are instantaneously aligned with $\mathcal{P}$ and $\mathcal{S}$ respectively, but stationary with respect to $\mathcal{W}$.

To simplify notation, let

$$
\begin{aligned}
\mathbf{R}_{1} & ={ }_{\mathcal{P}}^{\mathcal{W}} \mathbf{R} \\
\mathbf{R}_{2} & ={ }_{\mathcal{S}}^{\mathcal{P}} \mathbf{R}=\left[\begin{array}{ccc}
\cos \theta & -\sin \theta & 0 \\
\sin \theta & \cos \theta & 0 \\
0 & 0 & 1
\end{array}\right] \\
\mathbf{R}_{3} & ={ }_{\mathcal{S}}{ }^{\prime} \mathbf{R}=\mathbf{I} \\
\boldsymbol{\omega}_{1} & ={ }^{\mathcal{W}} \boldsymbol{\omega}_{\mathcal{P} / \mathcal{W}} \\
\boldsymbol{\omega}_{2} & ={ }^{\mathcal{P}} \boldsymbol{\omega}_{\mathcal{S} / \mathcal{P}}=[0,0, \dot{\theta}]^{T} \\
\boldsymbol{\omega}_{3} & ={ }^{\mathcal{S}^{\prime}} \boldsymbol{\omega}_{\mathcal{S} / \mathcal{S}^{\prime}},
\end{aligned}
$$

where $\theta$ is the orientation of the part with respect to the $x$-axis of the $\mathcal{P}$ frame and $\mathbf{I}$ is the $3 \times 3$ identity matrix.

Differentiating (1)-(3) yields

$$
\begin{aligned}
& \dot{\mathbf{R}}_{1}=\widehat{\boldsymbol{\omega}}_{1} \mathbf{R}_{1} \\
& \dot{\mathbf{R}}_{2}=\widehat{\boldsymbol{\omega}}_{2} \mathbf{R}_{2} \\
& \dot{\mathbf{R}}_{3}=\widehat{\boldsymbol{\omega}}_{3},
\end{aligned}
$$

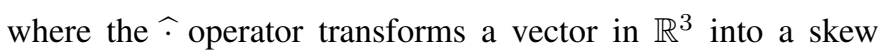
symmetric matrix in so(3) allowing cross products to be written as matrix multiplications.

To further simplify notation, vectors written without presuperscripts obey the following conventions.

- $\mathbf{p}, \dot{\mathbf{p}}, \ddot{\mathbf{p}}, \boldsymbol{\omega}_{1}$, and $\dot{\boldsymbol{\omega}}_{1}$ are expressed in $\mathcal{W}$ coordinates.

- $\mathbf{s}, \dot{\mathrm{s}}, \ddot{\mathrm{s}}, \boldsymbol{\omega}_{2}$, and $\dot{\omega}_{2}$ are expressed in $\mathcal{P}$ coordinates.

- $\boldsymbol{\omega}_{3}$ and $\dot{\boldsymbol{\omega}}_{3}$ are expressed in $\mathcal{S}^{\prime}$ coordinates.

- $\mathbf{c}_{1}, \mathbf{c}_{2}$, and $\mathbf{c}_{3}$ are expressed in $\mathcal{S}$ coordinates.

- All forces are expressed in $\mathcal{P}^{\prime}$ coordinates.

\section{DYNAMICS}

In this section we derive the dynamic equations governing an arbitrary rigid part in three-point frictional sliding contact with a moving rigid surface. Let $\mathbf{q}=\left(\mathbf{q}_{\text {plate }}, \mathbf{q}_{\text {part }}\right) \in Q$ specify the configuration of the full plate-part system such that $\mathbf{q}_{\text {plate }}=$ $\left(\mathbf{p}, \mathbf{R}_{1}\right) \in S E(3)$ represents the configuration of the plate 
with respect to $\mathcal{W}$ and $\mathbf{q}_{\text {part }}=\left(\mathbf{s}, \mathbf{R}_{2}\right) \in S E(3)$ represents the configuration of the part with respect to $\mathcal{P}$.

\section{A. Contact Constraints}

We make the following assumptions about the plate-part interface.

1) Three distinct points fixed to the part, $c_{1}, c_{2}$, and $c_{3}$, are always in contact with three points on the plate surface, $c_{1}^{*}, c_{2}^{*}$, and $c_{3}^{*}$. No other points on the part make contact with the plate.

2) $c_{1}, c_{2}$, and $c_{3}$ are noncollinear.

3) The velocity of $c_{i}$ does not equal the velocity of $c_{i}^{*}$ (i.e., $c_{1}, c_{2}$, and $c_{3}$ are always sliding on the plate).

4) The friction forces at the contact points are governed by Coulomb's law with kinetic friction coefficients $\mu_{k_{1}}$, $\mu_{k_{2}}$, and $\mu_{k_{3}}$.

As a consequence of assumption 1 , the $z$-component of $\mathbf{s}$ is fixed and $\mathbf{R}_{2}$ can be expressed in terms of the single parameter $\theta$. Thus, we now represent the part's configuration as $\mathbf{q}_{\text {part }}=$ $\left(s_{x}, s_{y}, \theta\right) \in \mathrm{SE}(2)$. Note that assumption 1 also implies the following constraints on accelerations relative to the $\mathcal{P}$ frame:

$$
\mathbf{e}_{z}^{T} \ddot{\mathbf{s}}=0, \quad \mathbf{e}_{x}^{T} \dot{\boldsymbol{\omega}}_{2}=0, \quad \mathbf{e}_{y}^{T} \dot{\boldsymbol{\omega}}_{2}=0,
$$

where $\mathbf{e}_{x}=[1,0,0]^{T}, \mathbf{e}_{y}=[0,1,0]^{T}$, and $\mathbf{e}_{z}=[0,0,1]^{T}$.

\section{B. Dynamic Model}

The dynamic model of the plate-part system is

$$
\dot{\mathbf{x}}=f(\mathbf{x}, \mathbf{u}, \boldsymbol{\sigma}), \quad \mathbf{x}(0)=\mathbf{x}_{0},
$$

where $\mathbf{x}=\left(\mathbf{x}_{\text {plate }}, \mathbf{x}_{\text {part }}\right) \in X$ is the state of the system such that $\mathbf{x}_{\text {plate }}=\left(\mathbf{p}, \mathbf{R}_{1}, \dot{\mathbf{p}}, \boldsymbol{\omega}_{1}\right) \in X_{\text {plate }}$ and $\mathbf{x}_{\text {part }}=\left(\mathbf{q}_{\text {part }}, \dot{\mathbf{q}}_{\text {part }}\right)=$ $\left(s_{x}, s_{y}, \theta, \dot{s}_{x}, \dot{s}_{y}, \dot{\theta}\right) \in X_{\text {part }}, \mathbf{u}=\left[\ddot{\mathbf{p}}^{T}, \dot{\boldsymbol{\omega}}_{1}^{T}\right]^{T} \in \mathbb{R}^{6}$ is the control input corresponding to the plate's acceleration with respect to $\mathcal{W}$, and $\boldsymbol{\sigma}=\left(m, \mathbf{J}, \mathbf{c}_{1}, \mathbf{c}_{2}, \mathbf{c}_{3}, \mu_{k_{1}}, \mu_{k_{2}}, \mu_{k_{3}}\right) \in \Sigma$ is the parameterization of the part where $m$ is its mass and

$$
\mathbf{J}=\left[\begin{array}{lll}
J_{x x} & J_{x y} & J_{x z} \\
J_{y x} & J_{y y} & J_{y z} \\
J_{z x} & J_{z y} & J_{z z}
\end{array}\right]
$$

is its symmetric positive definite rotational inertia matrix relative to $s$ and expressed in the $\mathcal{S}$ frame.

To numerically integrate (11) forward in time, we note that all elements of $\dot{\mathbf{x}}$ are known except $\ddot{\mathbf{q}}_{\text {part }}$ (i.e., $\dot{\mathbf{p}}$ and $\dot{\mathbf{q}}_{\text {part }}$ are elements of $\mathbf{x}, \dot{\mathbf{R}}_{1}$ is given by (7), and $\ddot{\mathbf{p}}$ and $\dot{\boldsymbol{\omega}}_{1}$ are elements of $\mathbf{u}$ ). The rest of this section is devoted to deriving $\ddot{\mathbf{q}}_{\text {part }}$.

\section{Force Balance}

In $\mathcal{P}^{\prime}$ coordinates, the gravitational force on the part is

$$
\mathbf{f}_{G}=-m g \mathbf{R}_{1}^{T} \mathbf{e}_{z}
$$

where ${ }^{\mathcal{W}} \mathbf{g}=[0,0,-g]^{T}$, and the normal and frictional forces at the $i$ th contact are

$$
\begin{aligned}
\mathbf{f}_{N_{i}} & =N_{i} \mathbf{e}_{z} \\
\mathbf{f}_{F_{i}} & =-\mu_{k_{i}} N_{i} \frac{\mathcal{P}^{\prime} \dot{\mathbf{c}}_{i}-{ }^{\mathcal{P}^{\prime}} \dot{\mathbf{c}}_{i}^{*}}{\left\|\mathcal{P}^{\prime} \dot{\mathbf{c}}_{i}-{ }^{\mathcal{P}^{\prime}} \dot{\mathbf{c}}_{i}^{*}\right\|},
\end{aligned}
$$

where $N_{i}>0$ is the magnitude of the normal force at the $i$ th contact, and ${ }^{\mathcal{P}^{\prime}} \dot{\mathbf{c}}_{i}-{ }_{\mathcal{P}^{\prime}} \dot{\mathbf{c}}_{i}^{*}={ }^{\mathcal{P}} \dot{\mathbf{c}}_{i}=\dot{\mathbf{s}}+\widehat{\boldsymbol{\omega}}_{2} \mathbf{R}_{2} \mathbf{c}_{i}$ is the sliding velocity of the $i$ th contact point. Note that $\left\|^{\mathcal{P}} \dot{\mathbf{c}}_{i}\right\|>0$ by assumption 3 ensuring that (14) is always well-defined.

Newton's second law for the linear dynamics of the part in the $\mathcal{P}^{\prime}$ frame is

$$
\mathbf{f}_{G}+\sum_{i=1}^{3} \mathbf{f}_{N_{i}}+\mathbf{f}_{F_{i}}=m^{\mathcal{P}^{\prime}} \ddot{\mathbf{s}} .
$$

Noting that ${ }^{\prime} \ddot{\mathbf{s}}=\mathbf{R}_{1}^{T \mathcal{W}} \ddot{\mathbf{s}}$ and using (12)-(14), this becomes

$$
-m g \mathbf{R}_{1}^{T} \mathbf{e}_{z}+\sum_{i=1}^{3} \mathbf{d}_{i} N_{i}=m \mathbf{R}_{1}^{T \mathcal{W}} \ddot{\mathbf{s}},
$$

where

$$
\mathbf{d}_{i}=\mathbf{e}_{z}-\mu_{k_{i}} \frac{\mathcal{P} \dot{\mathbf{c}}_{i}}{\left\|{ }^{\mathcal{P}} \dot{\mathbf{c}}_{i}\right\|} .
$$

Differentiating ${ }^{\mathcal{W}} \mathbf{s}=\mathbf{p}+\mathbf{R}_{1} \mathbf{s}$ twice with respect to time and substituting the result into (16) yields

$$
-m \ddot{\mathbf{s}}+\sum_{i=1}^{3} \mathbf{d}_{i} N_{i}=\mathbf{f}_{\dagger},
$$

where

$$
\mathbf{f}_{\dagger}=m \mathbf{R}_{1}^{T}\left(\ddot{\mathbf{p}}+\widehat{\boldsymbol{\omega}}_{1} \widehat{\boldsymbol{\omega}}_{1} \mathbf{R}_{1} \mathbf{s}+\widehat{\dot{\boldsymbol{\omega}}}_{1} \mathbf{R}_{1} \mathbf{s}+2 \widehat{\boldsymbol{\omega}}_{1} \mathbf{R}_{1} \dot{\mathbf{s}}+g \mathbf{e}_{z}\right) .
$$

Finally, we write (18) as

$$
-m \ddot{\mathbf{s}}+\mathbf{D n}=\mathbf{f}_{\dagger},
$$

where $\mathbf{D}=\left[\mathbf{d}_{1}, \mathbf{d}_{2}, \mathbf{d}_{3}\right] \in \mathbb{R}^{3 \times 3}$, and $\mathbf{n}=\left[N_{1}, N_{2}, N_{3}\right]^{T} \in$ $\mathbb{R}^{3}$.

\section{Torque Balance}

Euler's equation for the rotational dynamics of the part in the $\mathcal{S}^{\prime}$ frame is

$$
\sum_{i=1}^{3} \widehat{\mathbf{c}}_{i} \mathbf{R}_{2}^{T}\left(\mathbf{f}_{F_{i}}+\mathbf{f}_{N_{i}}\right)=\mathbf{J} \dot{\boldsymbol{\omega}}_{3}+\widehat{\boldsymbol{\omega}}_{3} \mathbf{J} \boldsymbol{\omega}_{3} .
$$

After substituting (12)-(14), we can write this as

$$
\mathbf{H n}=\mathbf{J} \dot{\boldsymbol{\omega}}_{3}+\widehat{\boldsymbol{\omega}}_{3} \mathbf{J} \boldsymbol{\omega}_{3}
$$

where $\mathbf{H}=\left[\mathbf{h}_{1}, \mathbf{h}_{2}, \mathbf{h}_{3}\right]$ and

$$
\mathbf{h}_{i}=\widehat{\mathbf{c}}_{i} \mathbf{R}_{2}^{T} \mathbf{d}_{i} .
$$

To rewrite $\boldsymbol{\omega}_{3}$ in terms of $\mathbf{x}$ we note that

$$
\begin{aligned}
\boldsymbol{\omega}_{3}={ }^{\mathcal{S}^{\prime}} \boldsymbol{\omega}_{\mathcal{S} / \mathcal{S}^{\prime}}= & { }^{\mathcal{S}^{\prime}} \boldsymbol{\omega}_{\mathcal{S} / \mathcal{P}}+{ }^{\mathcal{S}^{\prime}} \boldsymbol{\omega}_{\mathcal{P} / \mathcal{W}}+{ }^{\mathcal{S}^{\prime}} \boldsymbol{\omega}_{\mathcal{W} / \mathcal{S}^{\prime}} \\
= & { }_{\mathcal{S}}^{\mathcal{S}^{\prime}} \mathbf{R}{ }_{\mathcal{P}} \mathbf{R}^{\mathcal{P}} \boldsymbol{\omega}_{\mathcal{S} / \mathcal{P}} \\
& \quad+{ }_{\mathcal{S}}^{\mathcal{S}^{\prime}} \mathbf{R}{ }_{\mathcal{P}}^{\mathcal{S}} \mathbf{R}_{\mathcal{P}} \mathbf{R}^{T} \mathcal{W}_{\boldsymbol{\omega}_{\mathcal{P}} \mathcal{W}}+0 \\
= & \mathbf{R}_{3} \mathbf{R}_{2}^{T} \boldsymbol{\omega}_{2}+\mathbf{R}_{3} \mathbf{R}_{2}^{T} \mathbf{R}_{1}^{T} \boldsymbol{\omega}_{1} \\
= & \boldsymbol{\omega}_{2}+\mathbf{R}_{2}^{T} \mathbf{R}_{1}^{T} \boldsymbol{\omega}_{1} .
\end{aligned}
$$

Differentiating (26) gives $\dot{\boldsymbol{\omega}}_{3}$ in terms of $\mathbf{x}$ and $\mathbf{u}$ :

$$
\dot{\boldsymbol{\omega}}_{3}=\dot{\boldsymbol{\omega}}_{2}-\mathbf{R}_{2}^{T} \widehat{\boldsymbol{\omega}}_{2} \mathbf{R}_{1} \boldsymbol{\omega}_{1}+\mathbf{R}_{2}^{T} \mathbf{R}_{1}^{T} \dot{\boldsymbol{\omega}}_{1}
$$


Substituting (27) and (28) into (22) and rearranging gives

$$
-\mathbf{J} \dot{\omega}_{2}+\mathbf{H n}=\boldsymbol{\tau}_{\dagger},
$$

where

$$
\begin{aligned}
\boldsymbol{\tau}_{\dagger}= & \mathbf{J}\left(-\mathbf{R}_{2}^{T} \widehat{\boldsymbol{\omega}}_{2} \mathbf{R}_{1}^{T} \boldsymbol{\omega}_{1}+\mathbf{R}_{2}^{T} \mathbf{R}_{1}^{T} \dot{\boldsymbol{\omega}}_{1}\right) \\
& +\left(\widehat{\boldsymbol{\omega}}_{2}+\widehat{\mathbf{R}_{2}^{T} \mathbf{R}_{1}^{T} \boldsymbol{\omega}_{1}}\right) \mathbf{J}\left(\boldsymbol{\omega}_{2}+\mathbf{R}_{2}^{T} \mathbf{R}_{1}^{T} \boldsymbol{\omega}_{1}\right) .
\end{aligned}
$$

\section{E. Part Acceleration During Sliding}

Noting the contact constraints given by (10), we can solve for $\ddot{\mathbf{q}}_{\text {part }}$ by combining (20) and (29) into a single matrix equation $\mathbf{A z}=\mathbf{b}$ :

$$
\underbrace{\left[\begin{array}{cc}
-\mathbf{M}^{\prime} & \mathbf{D} \\
-\mathbf{J}^{\prime} & \mathbf{H}
\end{array}\right]}_{\mathbf{A} \in \mathbb{R}^{6 \times 6}} \underbrace{\left[\begin{array}{c}
\ddot{\mathbf{q}}_{\text {part }} \\
\mathbf{n}
\end{array}\right]}_{\mathbf{z} \in \mathbb{R}^{6 \times 1}}=\underbrace{\left[\begin{array}{c}
\mathbf{f}_{\dagger} \\
\boldsymbol{\tau}_{\dagger}
\end{array}\right]}_{\mathbf{b} \in \mathbb{R}^{6 \times 1}},
$$

where $\mathbf{M}^{\prime}=m\left(\mathbf{e}_{x} \mathbf{e}_{x}^{T}+\mathbf{e}_{y} \mathbf{e}_{y}^{T}\right)$ and $\mathbf{J}^{\prime}=\mathbf{J} \mathbf{e}_{z} \mathbf{e}_{z}^{T}$.

Note that $\mathbf{A}$ depends only on $\mathbf{x}$, and that $\mathbf{b}$ depends only on $\mathbf{x}$ and $\mathbf{u}$. Thus, given the current values of $\mathbf{x}$ and $\mathbf{u}$, there exists a unique solution for $\ddot{\mathbf{q}}_{\text {part }}$ as long as $\mathbf{A}$ is invertible. A sufficient condition for $\mathbf{A}$ to be invertible is noncollinearity of the three contact points (assumption 2), but we omit the proof here due to space constraints.

\section{F. Full Dynamics}

To simulate the full dynamics we numerically integrate (11) by solving (31) at each time step. Though guaranteed to exist, the solution to (31) is physically meaningless if one or more of the components of $\mathbf{n}$ is negative (indicating that contact was lost). If this occurs during a simulation, the simulation is terminated.

\section{G. U-Dynamics}

We now consider only the space of control signals $U$ that correspond to plate motions with the following properties:

1) Periodic: $\mathbf{p}(t+T)=\mathbf{p}(t), \mathbf{R}_{1}(t+T)=\mathbf{R}_{1}(t)$.

2) Small amplitude: $\|\mathbf{p}(t)\|<\epsilon_{1},\left\|\mathbf{R}_{1}(t)-\mathbf{I}\right\|<\epsilon_{2}$, such that $\epsilon_{1}$ and $\epsilon_{2}$ are sufficiently small to use the approximations $\mathbf{p}(t) \approx \mathbf{0}$ and $\mathbf{R}_{1}(t) \approx \mathbf{I}$ for all time.

3) Differentiable with respect to time: $\mathbf{p}, \mathbf{R}_{1} \in C^{1}$.

4) Induced Coriolis and centripetal part accelerations are negligible: terms in (19) and (30) composed of products of elements in $\mathbf{x}$ and $\mathbf{u}$ can be approximated as zero.

Equation (31) then simplifies to

$$
\mathbf{A} \mathbf{z}=\tilde{\mathbf{b}}=\left[\tilde{\mathbf{f}}_{\dagger}^{T}, \tilde{\boldsymbol{\tau}}_{\dagger}^{T}\right]^{T},
$$

where

$$
\begin{aligned}
\tilde{\mathbf{f}}_{\dagger} & =m\left(\ddot{\mathbf{p}}+\widehat{\boldsymbol{\omega}}_{1} \mathbf{s}_{0}+g \mathbf{e}_{z}\right) \\
\tilde{\boldsymbol{\tau}}_{\dagger} & =\mathbf{J R}_{2}^{T} \dot{\boldsymbol{\omega}}_{1} .
\end{aligned}
$$

To simulate $U$-dynamics, we numerically integrate (11) by solving (32) at each time step.

\section{H. Reduced U-Dynamics}

If we further assume that changes in the part's configuration are negligible over small time intervals (i.e., several periods of $\mathbf{u}(t) \in U$ ), then we refer to the dynamics as reduced $U$ dynamics. In this case, $\mathbf{q}_{\text {part }}(t)=\mathbf{q}_{\text {part }_{0}}=\left(s_{x_{0}}, s_{y_{0}}, \theta_{0}\right) \in$ $Q_{\text {part }_{0}}$ is any fixed part configuration with respect to $\mathcal{P}$. With this assumption, for any $\mathbf{q}_{\text {part }} \in Q_{\text {part }_{0}}$, the system evolves according to

$$
\dot{\tilde{\mathbf{x}}}=f(\tilde{\mathbf{x}}, \mathbf{u}, \boldsymbol{\sigma}), \quad \tilde{\mathbf{x}}(0)=\tilde{\mathbf{x}}_{0},
$$

where $\tilde{\mathbf{x}}=\left(\tilde{\mathbf{x}}_{\text {plate }}, \tilde{\mathbf{x}}_{\text {part }}\right)$ is the reduced state of the system such that $\tilde{\mathbf{x}}_{\text {plate }}=\left[\dot{\mathbf{p}}^{T}, \boldsymbol{\omega}_{1}^{T}\right]^{T}$ and $\tilde{\mathbf{x}}_{\text {part }}=\dot{\mathbf{q}}_{\text {part }} \in \tilde{X}_{\text {part }}=\mathbb{R}^{3}$. For this system, the initial state of the part $\tilde{\mathbf{x}}_{\text {part }}$ is arbitrary, but $\tilde{\mathbf{x}}_{\text {plate }_{0}}$ is uniquely specified by $\mathbf{u}(t)$ due to property 1 of signals in $U$. In particular, property 1 requires that $\tilde{\mathbf{x}}_{\text {plate }_{0}}$ satisfy $\int_{0}^{T} \tilde{\mathbf{x}}_{\text {plate }}(t) d t=\int_{0}^{T}\left(\tilde{\mathbf{x}}_{\text {plate }_{0}}+\int_{0}^{t} \mathbf{u}(\tau) d \tau\right) d t=\mathbf{0}$.

To simulate reduced $U$-dynamics we numerically integrate (35) by solving (32) at each time step.

\section{Asymptotic Twist Fields}

\section{A. Limit Cycles for Reduced U-Dynamics}

In general, the trajectory of $\mathbf{x}_{\text {part }}$ through $X_{\text {part }}$ depends on the initial state of the entire part-plate system. However, for reduced $U$-dynamics we observed the following property in simulations:

Observation 1: Given any part $\sigma \in \Sigma$, periodic input signal $\mathbf{u}(t) \in U$, and part configuration $\mathbf{q}_{\text {part }_{0}} \in Q_{\text {part }_{0}}$, the trajectory of $\tilde{\mathbf{x}}_{\text {part }}$ in $\tilde{X}_{\text {part }}$ converges to a unique stable limit cycle trajectory $\tilde{\mathbf{x}}_{\text {part }}^{\mathrm{LC}}(t)$ with period $T$, regardless of the part's initial state $\tilde{\mathbf{x}}_{\text {part }_{0}}$.

To illustrate Observation 1, we randomly generated a part, control signal, and configuration. Fig. 3(a) shows the trajectories of $\tilde{\mathbf{x}}_{\text {part }}$ for the two different initial states (solid red and blue circles) over a time interval of ten periods of $\mathbf{u}(t)$. Open circles are plotted in intervals of $T$ along both trajectories. Both trajectories rapidly converge to the same limit cycle trajectory $\tilde{\mathbf{x}}_{\text {part }}^{\mathrm{LC}}(t)$, which is plotted with a thick black line for clarity.

In the Appendix we outline a proof of the following special case of Observation 1:

Theorem 1: Assume a system with reduced $U$-dynamics. Given an infinitesimally thick part $\sigma \in \Sigma$ with all mass distributed in the plane spanned by the three contact points (i.e., the $x-y$ plane of $\mathcal{P}^{\prime}$ ), input signal $\mathbf{u}(t) \in U$, and part configuration $\mathbf{q}_{\text {part }_{0}} \in Q_{\text {part }_{0}}$, the trajectory of $\tilde{\mathbf{x}}_{\text {part }}$ in $\tilde{X}_{\text {part }}$ converges asymptotically to a unique limit cycle trajectory $\tilde{\mathbf{x}}_{\text {part }}^{\mathrm{LC}}(t)$ with period $T$, regardless of the part's initial state $\tilde{\mathbf{x}}_{\text {part }_{0}}$.

Theorem 1 generalizes the analysis in [11] that assumed the part was a point mass.

\section{B. Asymptotic Twists and Fields}

Observation 1 is significant because for any $\sigma \in \Sigma$ and $\mathbf{u}(t) \in U$, every part configuration $\mathbf{q}_{\text {part }}$ is associated with a unique trajectory $\tilde{\mathbf{x}}_{\text {part }}^{\mathrm{LC}}(t)$ in $\tilde{X}_{\text {part }}$. We define the asymptotic 


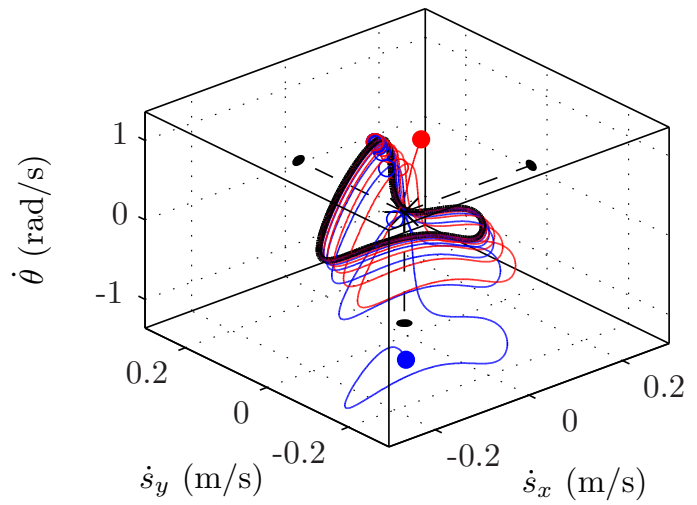

(a)

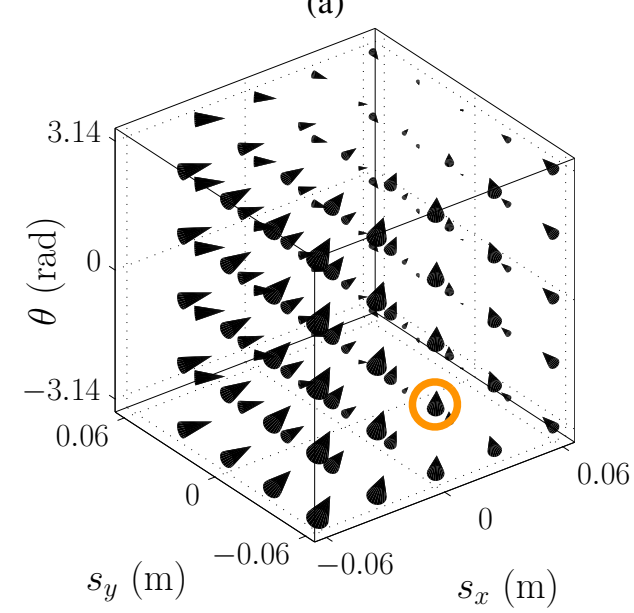

(b)

Fig. 3. Simulation data for a randomly generated plate motion and part. (a): The part is given two different initial states (filled blue and red circles) at the same configuration. Both initial states converge to the same limit cycle trajectory (thick black curve). Unfilled circular markers are plotted along the trajectories at intervals of $T$. The black circles on the side walls are projections of the asymptotic twist (i.e., time-averaged value of the limit cycle). (b): Vectors in the asymptotic twist field at approximately 125 configurations. The vector circled in orange is associated with the limit cycle/asymptotic twist in (a).

twist $\boldsymbol{\xi}_{a}$ at $\mathbf{q}_{\text {part }_{0}}$ as the time-averaged value of $\tilde{\mathbf{x}}_{\text {part }}^{\mathrm{LC}}(t)$ over one cycle of $\mathbf{u}(t)$ :

$$
\boldsymbol{\xi}_{a}\left(\mathbf{q}_{\mathrm{part}_{0}}\right)=\frac{1}{T} \int_{0}^{T} \tilde{\mathbf{x}}_{\text {part }}^{\mathrm{LC}}(t) d t .
$$

The asymptotic twist is undefined at $\mathbf{q}_{\text {part }}$ if the part loses contact during the cycle.

The asymptotic twist field corresponding to $(\boldsymbol{\sigma}, \mathbf{u}(t))$ is a map from $Q_{\text {part }_{0}}$ to the tangent bundle of $\operatorname{SE}(2)$, as defined by (36). Fig. 3(b) shows a portion of the asymptotic twist field for the same randomly generated part and control signal discussed in Section IV-A. The asymptotic twist vector circled in orange corresponds to the configuration associated with the limit cycle shown in Fig. 3(a).

\section{Asymptotic Twist Fields vs. Full Dynamic Simulations}

Asymptotic twists are only defined for systems with reduced $U$-dynamics; however, after an initial transient, the trajectory of $\mathbf{q}_{\text {part }}$ obtained from a full dynamic simulation is closely approximated by an integral curve of the corresponding asymptotic twist field. Figs. 1 and 4 correspond to the part

$$
\begin{array}{r}
\boldsymbol{\sigma}=\left(0.0216, \operatorname{diag}\left(1.084 * 10^{-5}, 0.123 * 10^{-5}, 1.20 * 10^{-5}\right),\right. \\
{[0.01,-0.03,-0.002]^{T},[0,0.03,-0.002]^{T},} \\
\left.[-0.01,-0.03,-0.002]^{T}, 0.3,0.3,0.3\right),
\end{array}
$$

and the respective plate motions

$$
\begin{aligned}
\mathbf{u}_{1}(t) & =[20 \sin (\omega t), 0,0,0,100 \sin (\omega t+3 \pi / 2), 0]^{T} \\
\mathbf{u}_{4}(t) & =[20 \sin (\omega t), 10 \sin (\omega t+\pi / 2), 0, \\
& 100 \sin (\omega t+549 \pi / 512), 100 \sin (\omega t+805 \pi / 512), 0]^{T},
\end{aligned}
$$

where $\omega=2 \pi / T$ and $T=1 / 20 \mathrm{~s}$ (all units in $\boldsymbol{\sigma}$ and $\mathbf{u}_{i}(t)$ are MKS). Each figure shows the part's trajectory through configuration space derived from a full dynamic simulation overlaid on the corresponding reduced $U$-dynamics asymptotic twist field computed at regularly spaced configurations.

\section{Stable Configurations and Sensorless Manipulation}

Asymptotic twist fields are particularly useful for sensorless manipulation planning. Points in the field with zero asymptotic twist correspond to fixed points in configuration space. Fields with stable fixed points can be used to position and orient parts without external vision sensors. For example, the field in Fig. 1 has lines of stable fixed points at $\left(0, s_{y}, 0\right)$ and $\left(0, s_{y}, \pi\right)$, and lines of unstable fixed points at $(0, y, \pi / 2)$ and $(0, y, 3 \pi / 2)$; the field in Fig. 4 has asymptotically stable fixed points at $(0,0,0)$ and $(0,0, \pi)$, and unstable fixed points at $(0,0, \pi / 2)$ and $(0,0,3 \pi / 2)$. Thus, regardless of the part's initial configuration, both of these fields almost surely orient the part to $\theta=0$ or $\theta=\pi$ as time evolves; the field in Fig. 4 also positions the part over the center of the plate, whereas the field in Fig. 1 positions it somewhere over the plate's $y$-axis.

\section{E. Sticking}

Our dynamic model assumes the part is perpetually sliding. By analyzing simulations, we found that this is reasonable for the part/plate motion combinations in which we are interested. In particular, sticking is generally avoided as long as $\mu_{k_{i}}<$ 0.5 and the plate motion is sinusoidal such that the peak accelerations of every point on the plate is at least $1-g$ in the horizontal plane. Of the fields presented in this paper, only the one in Fig. 6 has configurations in which sticking occurs during a portion of the cycle. Since the acceleration in the horizontal plane is zero at the center of the plate for this particular plate motion ( see $\mathbf{u}_{6}(t)$ in Section $\mathrm{V}$ ), it is not surprising that the sticking configurations are the ones in which a contact point is near the center of the plate.

\section{EXPERIMENTAL RESUlts}

Experimental data was collected using a camera running at 10 frames per second mounted directly above our 6-DoF parallel manipulator. The plate surface was covered with glass. The part was a rectangular aluminum block. Three $2 \mathrm{~mm}$ diameter steel spheres were glued to one side of the part 


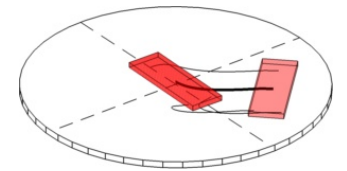

(a)

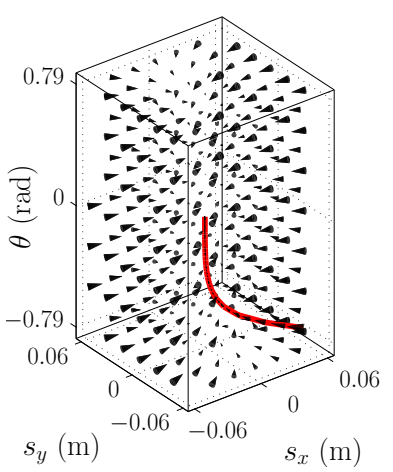

(b)

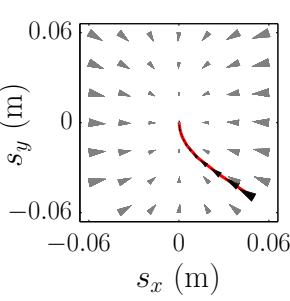

(c)

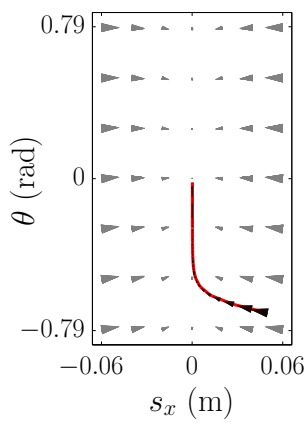

(d)

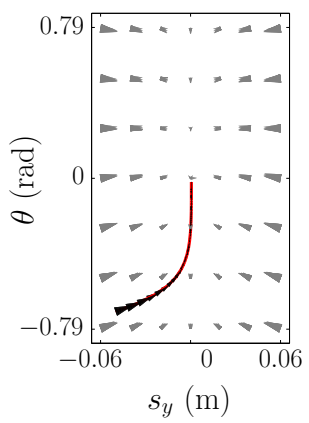

(e)

Fig. 4. Simultaneously orienting and positioning a part without sensors in simulation. (a): Full dynamic simulation (20 seconds) for $\boldsymbol{\sigma}$ and $\mathbf{u}_{4}(t)$ given in Section IV-C. The part is initially at rest near the edge of the plate and moves to a stable configuration with its center of mass over the center of the plate and its principal axes aligned with the $x$ - and $y$-axes of the plate. The thicker line denotes the path of the center of mass; the thinner lines denote the paths of the part's contact points. (b): Simulation in (a) represented as a trajectory through configuration space (red line) overlaid on the corresponding asymptotic twist field. (c)-(e): Trajectory and field vectors in (b) projected onto $s_{x}-s_{y}, s_{x}-\theta$, and $s_{y}-\theta$ planes. Black field vectors are plotted at configurations along the simulated trajectory every 0.25 seconds. Gray field vectors are projections of the 343 spatially distributed vectors in (b). Zoom in for more detail.

to create three-point contact with the plate. The full part description is given by

$$
\begin{array}{r}
\boldsymbol{\sigma}=\left(0.0539, \operatorname{diag}\left(4.90 * 10^{-5}, 2.18 * 10^{-6}, 5.04 * 10^{-5}\right),\right. \\
{[0,0.052,-0.006]^{T},[-0.01,-0.052,-0.006]^{T},} \\
\left.[0.01,-0.052,-0.006]^{T}, 0.17,0.17,0.17\right) .
\end{array}
$$

Figs. 5 and 6 show data for the respective plate motions

$$
\begin{aligned}
\mathbf{u}_{5}(t) & =[14 \sin (\omega t), 7 \sin (\omega t+\pi / 2), \\
& 5 \sin (\omega t+\pi / 20), 0,100 \sin (\omega t+5 \pi / 3), 0]^{T} \\
\mathbf{u}_{6}(t) & =[0,0,5 \sin (\omega t), 0,0,150 \sin (\omega t+3 \pi / 2)]^{T},
\end{aligned}
$$

where $\omega=2 \pi / T$ and $T=1 / 20 \mathrm{~s}$ (all units in $\boldsymbol{\sigma}$ and $\mathbf{u}_{i}(t)$ are MKS). Each figure shows the part's trajectory through configuration space overlaid on the corresponding reduced $U$ dynamics asymptotic twist field computed at regularly spaced configurations.

The field in Fig. 5 has no fixed points, but the sets of configurations given by $\left(0, s_{y}, 0\right)$ and $\left(0, s_{y}, \pi\right)$ are stable attractors (the sets of configurations given by $\left(0, s_{y}, \pi / 2\right)$ and $\left(0, s_{y}, 3 \pi / 2\right)$ are repellors). Consequently, the field continuously reduces uncertainty in the part's orientation while simultaneously transporting it in the $y$-direction. The field in Fig. 6 rotates the part. It has no fixed points.

In experiments, the exact motion of the part changes somewhat from trial to trial, most likely due to variations in the friction coefficient. See [11] for typical statistics.

\section{CONCLUSION}

We have shown that the motion of a rigid object sliding on a periodically vibrating rigid plate is well described by an asymptotic twist field. Future work will aim to find closed form solutions for certain classes of asymptotic twist fields as functions of $\mathbf{u}(t)$ and $\boldsymbol{\sigma}$, as was done with asymptotic velocity fields in [12]. This will lead to a better understanding of what types of fields can be generated. We are also studying the related problem: given a part $\boldsymbol{\sigma}$ and a desired final configuration $\mathbf{q}_{\text {part }}$, efficiently find a control signal $\mathbf{u}(t)$ that generates a field with $\mathbf{q}_{\text {part }}$ as the only stable fixed point. Currently, to find $\mathbf{u}(t)$ we must run a costly optimization that sheds little light on the relationship between $\mathbf{u}(t), \boldsymbol{\sigma}$, and the resulting asymptotic twist field.

\section{APPENDIX}

Here we outline a proof of Theorem 1, which states that for reduced $U$-dynamics, the state of a part with infinitesimal thickness asymptotically converges to a unique stable limit cycle.

\section{A. Decoupled, State-Independent Normal Forces}

The constraint on the mass distribution implies that each contact point $\mathbf{c}_{i}$ has the form $\left[c_{i, x}, c_{i, y}, 0\right]^{T}$, and that $J_{x z}=$ $J_{y z}=J_{z x}=J_{z y}=0$ in the part's rotational inertia matrix J. As a result, the A matrix in (32) simplifies in two ways. First, $\mathbf{J}^{\prime}$ has the form $\mathbf{e}_{z}^{T} \mathbf{e}_{z} \mathbf{J} \mathbf{e}_{z}^{T} \mathbf{e}_{z}$, and second, the columns of $\mathbf{H}$ have the form $\mathbf{h}_{i}=\left[c_{i, y},-c_{i, x}, h_{i, z}\right]$. Writing out (32) explicitly with these simplifications gives

$$
\left[\begin{array}{cccccc}
-m & 0 & 0 & d_{1, x} & d_{2, x} & d_{3, x} \\
0 & -m & 0 & d_{1, y} & d_{2, y} & d_{3, y} \\
0 & 0 & 0 & 1 & 1 & 1 \\
0 & 0 & 0 & c_{1, y} & c_{2, y} & c_{3, y} \\
0 & 0 & 0 & -c_{1, x} & -c_{2, x} & -c_{3, x} \\
0 & 0 & -J_{z z} & h_{1, z} & h_{2, z} & h_{3, z}
\end{array}\right]\left[\begin{array}{c}
\ddot{s}_{x} \\
\ddot{s}_{y} \\
\ddot{\theta} \\
N_{1} \\
N_{2} \\
N_{3}
\end{array}\right]=\tilde{\mathbf{b}} .
$$

By swapping rows three and six, A becomes block diagonal indicating that the normal forces $\mathbf{n}$ decouple from the part accelerations $\ddot{\mathbf{q}}_{\text {part }}$. After swapping, the bottom three rows can be solved independently to compute the normal forces:

$$
\left[\begin{array}{ccc}
c_{1, y} & c_{2, y} & c_{3, y} \\
-c_{1, x} & -c_{2, x} & -c_{3, x} \\
1 & 1 & 1
\end{array}\right]\left[\begin{array}{c}
N_{1} \\
N_{2} \\
N_{3}
\end{array}\right]=\left[\begin{array}{c}
\mathbf{e}_{x}^{T} \mathbf{J R}_{2}^{T} \dot{\boldsymbol{\omega}}_{1} \\
\mathbf{e}_{y}^{T} \mathbf{J R}_{2}^{T} \dot{\boldsymbol{\omega}}_{1} \\
\mathbf{e}_{z}^{T} m\left(\ddot{\mathbf{p}}+\widehat{\dot{\boldsymbol{\omega}}}_{1} \mathbf{s}_{0}+g \mathbf{e}_{z}^{T}\right)
\end{array}\right] .
$$

The significance of (38) is that the normal forces are independent of sliding velocities at the contacts, and therefore 


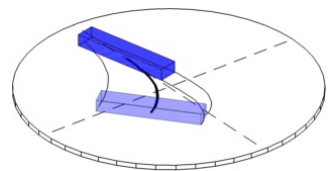

(a)

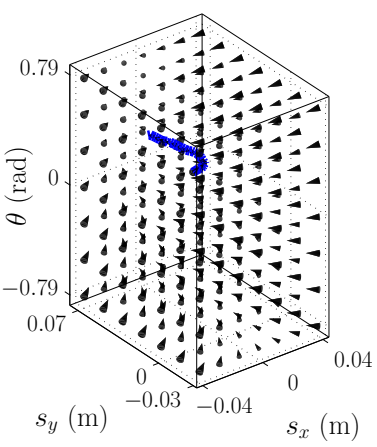

(b)

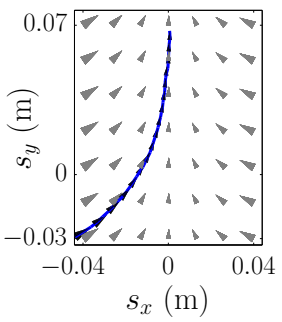

(c)

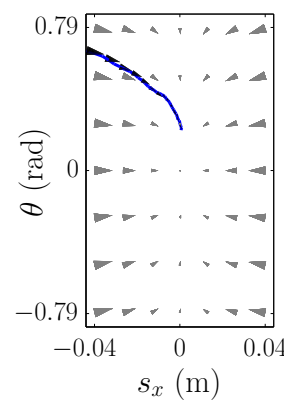

(d)

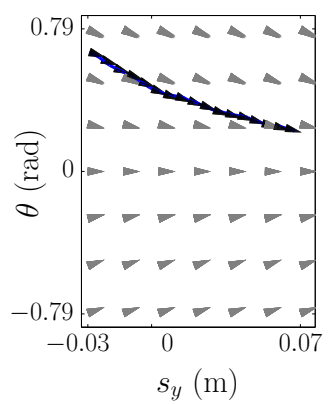

(e)

Fig. 5. Simultaneously transporting and orienting a part without sensors in experiment. (a): Reconstructed motion from camera data (4 seconds) for $\sigma$ and $\mathbf{u}_{5}(t)$ given in Section V. The thicker line denotes the path of the center of mass; the thinner lines denote the paths of the part's contact points. (b): Experimental data in (a) represented as a trajectory through configuration space (blue line) overlaid on the corresponding asymptotic twist field. (c)-(e): Trajectory and field vectors in (b) projected onto $s_{x}-s_{y}, s_{x}-\theta$, and $s_{y}-\theta$ planes. Black field vectors are plotted at configurations along the experimental trajectory every 0.25 seconds. Gray field vectors are projections of the 343 spatially distributed vectors in (b). Zoom in for more detail.

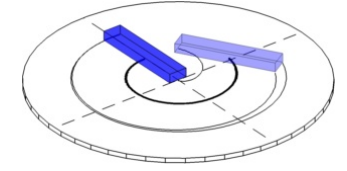

(a)

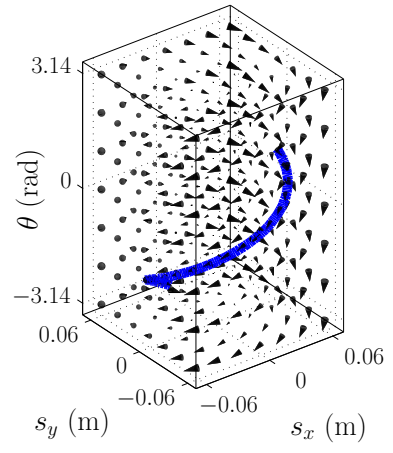

(b)

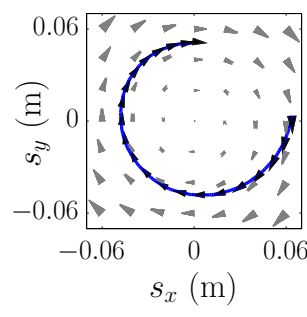

(c)

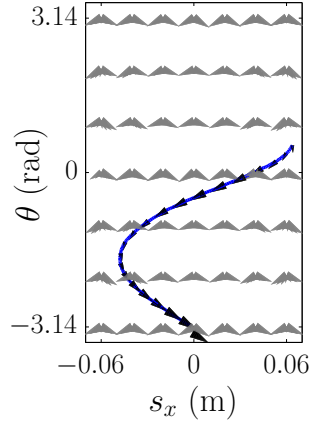

(d)

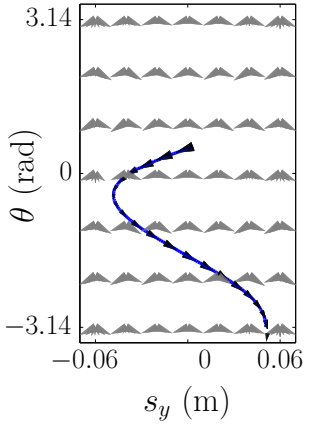

(e)

Fig. 6. Rotating a part in experiment. (a): Reconstructed motion from camera data ( 20 seconds) for $\boldsymbol{\sigma}$ and $\mathbf{u}_{6}(t)$ given in Section V. The thicker line denotes the path of the center of mass; the thinner lines denote the paths of the part's contact points. (b): Experimental data in (a) represented as a trajectory through configuration space (blue line) overlaid on the corresponding asymptotic twist field. (c)-(e): Trajectory and field vectors in (b) projected onto $s_{x}-s_{y}, s_{x}-\theta$, and $s_{y}-\theta$ planes. Black field vectors are plotted at configurations along the experimental trajectory every 1 second. Gray field vectors are projections of the 343 spatially distributed vectors in (b). Zoom in for more detail.

independent of $\tilde{\mathbf{x}}_{\text {part }}$ (i.e., $\dot{\mathbf{s}}$ and $\boldsymbol{\omega}_{2}$ ). This is only true due to the constraint that all the part's mass is distributed in the contact plane; in general, the normal force at each contact depends on the direction of $\tilde{\mathbf{x}}_{\text {part }}$ and is also coupled to $\ddot{\mathbf{q}}_{\text {part }}$.

\section{B. Limit Surfaces}

Since the normal forces are independent of $\tilde{\mathbf{x}}_{\text {part }}$, the part dynamics at each instant can be described using a frictional limit surface, as developed in [14], [15]. Limit surfaces provide a geometric method of relating a part's twist relative to the plate to the frictional wrench the plate exerts on the part. ${ }^{1}$ The part's limit surface is defined as the boundary of a set in wrench space whose elements correspond to all frictional wrenches that the part can exert on the plate. If the friction law at each contact point is dissipative and obeys the maximum work inequality [14] (such as Coulomb friction), then the limit

\footnotetext{
${ }^{1}$ Throughout the rest of the appendix we define a twist $\boldsymbol{\xi}=\left[\xi_{x}, \xi_{y}, \xi_{\theta}\right]^{T} \in$ $\mathbb{R}^{3}$ such that $\xi_{x}$ and $\xi_{y}$ are the linear $x$ and $y$ velocities, and $\xi_{\theta}$ is the angular velocity multiplied by the part's radius of gyration $\rho=\sqrt{J_{z z} / m}$. Similarly, we define a wrench $\mathbf{w}=\left[w_{x}, w_{y}, w_{\theta}\right]^{T} \in \mathbb{R}^{3}$ such that $w_{x}$ and $w_{y}$ are the $x$ and $y$ forces, and $w_{\theta}$ is the torque divided by $\rho$. This allows twists to live in a linear velocity space and wrenches to live in a force space.
}

surface is compact, convex, and encloses the origin of the wrench space. If the relative twist between the part and the plate is nonzero, then the frictional wrench the part exerts on the plate corresponds to a point $w$ on the limit surface. The vector from $w$ to the origin represents the frictional wrench exerted on the part by the plate. The key property of the limit surface, which arises from the maximum work inequality, is that the relative twist vector is normal to the limit surface at $w$ (assuming there is a well-defined normal). These ideas are illustrated in Fig. 7 for the simpler to visualize situation in which there are no angular terms (e.g., the part is a point mass and twists and wrenches are measured with respect to the center of mass).

In general, a limit surface may have flat faces (where a single twist direction maps to multiple wrenches) and vertices (where a single wrench maps to multiple twists due to lack of a well-defined normal vector). However, we have already stated that at each instant during sliding the part has a unique value of $\ddot{\mathbf{q}}_{\text {part }}$ (and therefore exerts a unique wrench) for any twist $\dot{\mathbf{q}}_{\text {part }}$. Consequently, the frictional wrench will never lie on a face during sliding. Additionally, limit surfaces of parts 


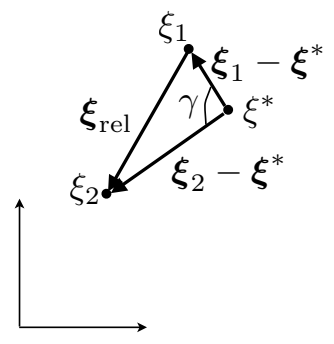

(a) Twist space

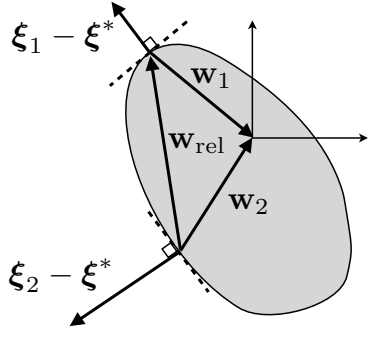

(b) Wrench space
Fig. 7. Two-dimensional analogues of full three-dimensional twist and wrench spaces. (a): Twist vectors relative to the $\mathcal{P}^{\prime}$ frame. (b): Limit surface (boundary of gray region) and wrench vectors relative to the $\mathcal{P}^{\prime}$ frame.

with noncollinear contact points do not contain vertices [14], ensuring the normal direction is always well-defined.

\section{Trajectory Convergence}

Assume the conditions of Theorem 1. Let two identical parts $\boldsymbol{\sigma}_{1}$ and $\boldsymbol{\sigma}_{2}$ have the same configuration $\mathbf{q}_{\text {part }_{0}}$, but arbitrary initial states. Let the twists $\boldsymbol{\xi}_{1}$ and $\boldsymbol{\xi}_{2}$ represent the state of the parts in the $\mathcal{P}^{\prime}$ frame at some instant, and the twist $\boldsymbol{\xi}^{*}$ represent the state of the plate in the $\mathcal{P}^{\prime}$ frame at the same instant (Fig. 7(a)). Note that the vector $\boldsymbol{\xi}_{i}-\boldsymbol{\xi}^{*}$ represents the relative twist of part $i$ with respect to the plate, and the vector $\boldsymbol{\xi}_{\text {rel }}=\boldsymbol{\xi}_{2}-\boldsymbol{\xi}_{1}$ represents the relative twist between the two parts. The wrenches exerted by the plate on the parts are denoted $\mathbf{w}_{1}$ and $\mathbf{w}_{2}$, and we define $\mathbf{w}_{\text {rel }}=\mathbf{w}_{2}-\mathbf{w}_{1}$ (Fig. 7(b)). Because of property 4 of $U$-dynamics and the way we have defined twists and wrenches (see previous footnote), $\dot{\boldsymbol{\xi}}_{\text {rel }}$ and $\mathbf{w}_{\text {rel }}$ are related by the following equation:

$$
\mathbf{w}_{\text {rel }}=m \dot{\boldsymbol{\xi}}_{\text {rel }} .
$$

We wish to show that $\boldsymbol{\xi}_{\text {rel }}=\mathbf{0}$ is globally asymptotically stable. Using the Lyapunov function $V=\frac{1}{2}\left\|\boldsymbol{\xi}_{\text {rel }}\right\|^{2}$, we can first show that $\boldsymbol{\xi}_{\text {rel }}$ is stable (i.e., the distance between $\boldsymbol{\xi}_{1}$ and $\boldsymbol{\xi}_{2}$ in twist space cannot increase).

Note that

$$
\begin{aligned}
\dot{V}=\boldsymbol{\xi}_{\mathrm{rel}} \cdot \dot{\boldsymbol{\xi}}_{\mathrm{rel}} & =\frac{1}{m} \boldsymbol{\xi}_{\mathrm{rel}} \cdot \mathbf{w}_{\mathrm{rel}} \\
& =\frac{1}{m}\left(\left(\boldsymbol{\xi}_{2}-\boldsymbol{\xi}^{*}\right) \cdot \mathbf{w}_{\mathrm{rel}}-\left(\boldsymbol{\xi}_{1}-\boldsymbol{\xi}^{*}\right) \cdot \mathbf{w}_{\mathrm{rel}}\right) .
\end{aligned}
$$

Because $\boldsymbol{\xi}_{i}-\boldsymbol{\xi}^{*}$ is normal to the limit surface and the limit surface is convex, it follows that $\left(\boldsymbol{\xi}_{2}-\boldsymbol{\xi}^{*}\right) \cdot \mathbf{w}_{\text {rel }} \leq 0$ and $\left(\boldsymbol{\xi}_{1}-\boldsymbol{\xi}^{*}\right) \cdot \mathbf{w}_{\text {rel }} \geq 0$ (see Fig. $7(\mathrm{~b})$ ). Thus, $\dot{V} \leq 0$, which implies that $\boldsymbol{\xi}_{\text {rel }}$ is stable.

For asymptotic stability, we must show that the system cannot indefinitely maintain a state where $\dot{V}=0$ and $V \neq 0$. Proving this relies on the fact that $\xi^{*}$ and $\dot{\xi}^{*}$ are periodic and bounded, but we omit the details here due to space constraints.

\section{Periodicity}

Given that any $\boldsymbol{\xi}_{1}$ and $\boldsymbol{\xi}_{2}$ converge to the same trajectory for each $\mathbf{q}_{\text {part }}$, we now prove that this trajectory is periodic

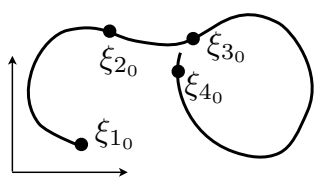

Fig. 8. Trajectories of $\boldsymbol{\xi}_{i}$ through twist space.

with period $T$. Consider any part trajectory $\boldsymbol{\xi}_{1}(t)$ beginning at $\boldsymbol{\xi}_{1_{0}}=\boldsymbol{\xi}_{1}(0)$. Let a second trajectory $\boldsymbol{\xi}_{2}(t)$ begin at $\boldsymbol{\xi}_{2_{0}}=$ $\boldsymbol{\xi}_{1}(T)$, and in general, an $i$ th trajectory $\boldsymbol{\xi}_{i}(t)$ begin at $\boldsymbol{\xi}_{i_{0}}=$ $\boldsymbol{\xi}_{1}\left((i-1) T\right.$ ) (Fig. 8). Because $\boldsymbol{\xi}^{*}$ is periodic with period $T$, $\boldsymbol{\xi}_{i}(t)=\boldsymbol{\xi}_{1}(t+(i-1) T)$; therefore Fig. 8 appears to have only a single curve emanating from $\boldsymbol{\xi}_{1_{0}}$. Because $\left\|\boldsymbol{\xi}_{\text {rel }}\right\| \rightarrow 0$ as $t \rightarrow 0$, it follows that $\left\|\boldsymbol{\xi}_{(i+1)_{0}}-\boldsymbol{\xi}_{i_{0}}\right\| \rightarrow 0$ as $i \rightarrow \infty$. It immediately follows that $\boldsymbol{\xi}_{1}$ converges to a periodic trajectory with period $T$.

\section{REFERENCES}

[1] K.-F. Böhringer, B. R. Donald, L. E. Kavraki, and F. Lamiraux, "A single universal force field can uniquely orient non-symmetric parts," in International Symposium on Robotics Research. Springer, 1999, pp. 395-402.

[2] K.-F. Böhringer, B. R. Donald, and N. C. MacDonald, "Programmable force fields for distributed manipulation, with applications to MEMS actuator arrays and vibratory parts feeders," International Journal of Robotics Research, vol. 18(2), pp. 168-200, Feb 1999.

[3] K.-F. Böhringer, L. Kavraki, and F. Lamiraux, "Part orientation with one or two stable equilibria using programmable force fields," IEEE Trans. Robot. Autom., vol. 16, pp. 157-170, Apr 2000.

[4] F. Lamiraux and L. Kavraki, "Positioning symmetric and non-symmetric parts using radial and constant force fields," in Workshop on the Algorithmic Foundations of Robotics, 2000.

[5] J. E. Luntz, W. Messner, and H. Choset, "Distributed manipulation using discrete actuator arrays," International Journal of Robotics Research, vol. 20 , no. 7 , July 2001 .

[6] A. Sudsang, "Sensorless sorting of two parts in the plane using programmable force fields," in IEEE International Conference on Intelligent Robots and Systems, 2002, pp. 1784-1789.

[7] T. Murphey and J. Burdick, "Feedback control for distributed manipulation systems that involve mechanical contacts," International Journal of Robotics Research, vol. 23, no. 7, pp. 763-781, Jul. 2004.

[8] K. Varsos, H. Moon, and J. Luntz, "Generation of quadratic potential force fields from flow fields for distributed manipulation," IEEE Transactions on Robotics, vol. 22, no. 1, pp. 108-118, Feb. 2006.

[9] D. Reznik and J. Canny, "C'mon part, do the local motion!" in IEEE International Conference on Robotics and Automation, 2001, pp. 22352242.

[10] M. Higashimori, K. Utsumi, Y. Omoto, and M. Kaneko, "Dynamic manipulation inspired by the handling of a pizza peel," in IEEE Transactions on Robotics, vol. 25, no. 4, 2009, pp. 829-838.

[11] T. H. Vose, P. Umbanhowar, and K. M. Lynch, "Friction-induced velocity fields for point parts sliding on a rigid oscillated plate," International Journal of Robotics Research, vol. 28, no. 8, pp. 1020-1039, 2009.

[12] _ , "Toward the set of frictional velocity fields generable by 6-degreeof-freedom motion of a rigid plate," in IEEE International Conference on Robotics and Automation, 2010.

[13] — "Friction-induced lines of attraction and repulsion for parts sliding on an oscillated plate," IEEE Transactions on Automation Science and Engineering, vol. 6, pp. 685-699, 2009.

[14] S. Goyal, A. Ruina, and J. Papadopoulos, "Planar sliding with dry friction: Part 1. limit surface and moment function," Wear, vol. 143, no. 2, pp. 307-330, 1991.

[15] — - "Planar sliding with dry friction: Part 2. dynamics of motion," Wear, vol. 143, no. 2, pp. 331-352, 1991. 